\title{
Engineering Proton Conductivity in Melanin Using Metal Doping
}

\author{
A. Bernardus Mostert*1, Shermiyah B. Rienecker ${ }^{2}$, Margarita Sheliakina $^{3}$, Paul Zierep ${ }^{4}$, \\ Graeme R. Hanson ${ }^{5}$, Jeffrey R. Harmer ${ }^{5}$, Gerhard Schenk ${ }^{6}$, Paul Meredith ${ }^{3,7}$
}

We dedicate this work to our friend and colleague Prof. Graeme Hanson, who passed away during the work done on this manuscript.

${ }^{1}$ Department of Chemistry, Swansea University, Singleton Park, SA2 8PP, Wales, UK

E-mail: a.b.mostert@swansea.ac.uk

${ }^{2}$ Biomedical Technology Services, Sunshine Coast University Hospital, Birtinya, QLD, 4575, Australia

${ }^{3}$ School of Mathematics and Physics, University of Queensland, Brisbane, St Lucia, QLD, 4072, Australia

${ }^{4}$ Institut für Physikalische Chemie, Albert-Ludwigs-Universität, Freiburg, Germany

${ }^{5}$ Centre of Advanced Imaging, University of Queensland, Brisbane, St Lucia, QLD, 4072, Australia

${ }^{6}$ School of Chemistry and Molecular Biosciences, University of Queensland, Brisbane, St Lucia, QLD, 4072, Australia

${ }^{7}$ Department of Physics, Swansea University, Singleton Park, SA2 8PP, Wales, UK

Keywords: bioelectronics, melanins, proton conductivity, doping, hydration control

\begin{abstract}
Long range electrical conduction in biomaterials is an increasingly active area of research, which includes systems such as the conductive pili, proteins, biomacromolecules, biocompatible conductive polymers and their derivatives. One material of particular interest, the human skin pigment melanin, is a long range proton conductor and recently demonstrated as capable of proton-to-electron transduction in a solid-state electrochemical transistor platform. In this work, a novel “doping strategy” is proposed to enhance and control melanin’s proton conductivity, potentially enhancing its utility as a transducing material. By chelating the transition metal ion $\mathrm{Cu}(\mathrm{II})$ into the bio-macromolecular matrix, free proton concentration and hence conductivity can be modulated. We confirm these observations by demonstrating enhanced performance in a next generation electrochemical transistor. Finally, the underlying mechanism is investigated via the use of a novel in-situ hydration-controlled Electron
\end{abstract}


Paramagnetic Resonance study, deducing that the enhanced proton concentration is due to controlling the internal solid-state redox chemistry of the intrinsic polyindolequinone structure. This doping strategy should be open to any transition metal ions that bind to hydroquinone systems (e.g. polydopamine). As such, the tailoring strategy could make other soft solid-state ionic systems more accessible to applications in bioelectronics, leading to the creation of higher performance ion-electron coupled devices.

\section{Introduction}

Harking back to the early 2000's with infamous claims of long range charge transport in DNA, ${ }^{1}$ 23 there has been continued interest in biomaterials that can conduct charges across relatively large distances (up to microns), ${ }^{4} 567891011$ in order to develop not only biocompatible conductors, but also to understand the fundamentals of such processes in nature. Microbial systems and their extracellular appendages which can extend up to tens of microns to enable anaerobic respiration are prime examples, c.f. Shewanella oneidensis and Geobacter sulfurreducens, ${ }^{5}$ with the latter having attracted attention for claims that its pili are essentially nanowires that conduct electrons via a "metallic-like" mechanism. ${ }^{12}$ The above observations have spawned a new sub field of bioelectronics in which peptide and protein analogues are being synthesised to determine if the electronic conductivity can be tuned by various mechanisms. $^{5}$

Long range ionic conduction in biomaterials is also of considerable interest and materials such as polysaccharides (e.g. cellulose, chitin and chitosan), proteins (e.g. bovine serum albumin) and polyindolequinones (e.g. eumelanin, polydopamine) are actively studied. ${ }^{9}$ Of particular interest is eumelanin, the brown-black pigment, which will be referred to as melanin for ease of nomenclature hereafter. ${ }^{13}$ Melanin has been shown to possess some remarkable and potentially useful electrical properties such as hydration dependent ionic conductivity, ${ }^{14} 151617$ 
1819 supercapacitance, ${ }^{20}$ and energy storage ${ }^{21}$. It has been utilised in elements in $\mathrm{pH}$ sensors ${ }^{22}$ and as a component in the first reported all-solid-state organic electrochemical transistor ${ }^{23}$ (OECT) in which it is used as a proton gate. The origin of the proton conductivity in melanin is not due to only basic acid/base dissociation chemistry (as for the archetypal synthetic proton conductor Nafion ${ }^{24}$ ), but it is also strongly coupled to the redox chemistry of its constituent polyindolequinone structure. ${ }^{25}$ A specific redox reaction, the comproportionation equilibrium (Figure 1B), drives the creation of protons and free radical species upon hydration, local $\mathrm{pH}$ change or illumination with UV and visible light.

A

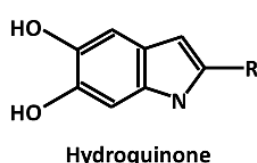

Hydroquinone

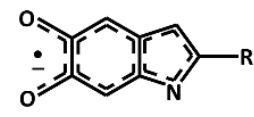

Semiquinone

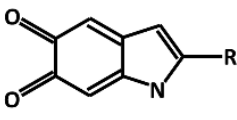

Quinone

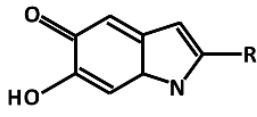

Quinone Imine
B

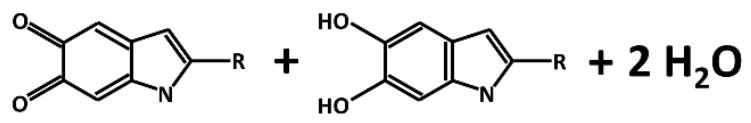

Quinone Hydroquinone $\mathrm{pk}_{\mathrm{a}} \|$

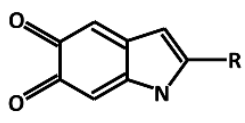

Quinone<smiles>[R]c1cc2cc(OC)c(O[13CH3])cc2[nH]1</smiles>

Deprotonated Hydroquinone

Redox,

SQ $\mid$

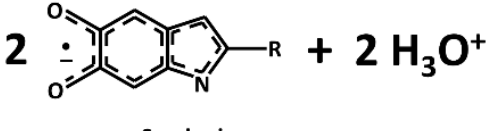

Figure 1. A) The starting monomer units for the melanin biopolymer. The top left monomer, when $\mathrm{R}$ is $\mathrm{H}$, is a dihydroxyindole (DHI). When $\mathrm{R}$ is $\mathrm{COOH}$, it is dihydroxyindole carboxylic acid (DHICA). The other monomers shown are various redox states of DHI/DHICA. B) The comproportionation equilibrium. ${ }^{25}$ The mechanism depicted was originally proposed by Chio et al. ${ }^{26}$ \& Felix $e t a l .{ }^{27}$ The reaction proceeds by the complete deprotonation of the hydroquinone, followed by a 1-electron transfer to the quinone moiety to form semiquinones. The reaction overall generates 2 hydroniums, or two protons available for conduction.

A detailed understanding of this redox chemistry, together with longstanding evidence that hydroquinones effectively bind transition metals at the catechol sites, 2728293031 has led us to 
postulate that doping with (for example) $\mathrm{Cu}$ (II) could be used to perturb the comproportionation equilibrium and hence control/modulate free proton density in the solid state. Whilst this is not strictly speaking 'doping' in the solid-state physics sense, similar strategies are represented as such in organic optoelectronics and so we loosely adopt the nomenclature in order to exemplify the outcome - i.e. engineered control of electrical properties via the addition of an extrinsic component.

In the work presented herein we study the effect of adding controlled amounts of $\mathrm{Cu}(\mathrm{II})$ ions into melanin. We choose $\mathrm{Cu}(\mathrm{II})$, since there is a wide body of literature already available showing it chelates in large quantities in melanin. ${ }^{28} 303132$ While the same is true for iron we selected copper in our study since it has only one apparent oxidative state (Cu(II)) when binding to melanin, ${ }^{29}$ whereas Fe may have a number of oxidative states, a fact that is likely to complicate the analysis of the role of metal ions in the conductivity of melanin. Having understood the 'doping effects' on basic material properties, we demonstrate a solid-state OECT based upon $\mathrm{Cu}(\mathrm{II})$-melanin films and show that long range proton conductivity is measurably enhanced in functioning proton-to-electron transducing devices. We complete the study by exploring the potential underlying mechanism of how $\mathrm{Cu}$ (II) changes the proton conductivity via an in-situ Electron Paramagnetic Resonance (EPR) hydration study, which allows us to determine the free radical spin concentrations.

\section{Experimental Section}

Material Synthesis: Melanin was synthesised following a standard literature procedure. ${ }^{27} 3334$ In brief, D,L-dopa (Sigma-Aldrich) was dissolved in deionised water, subsequently adjusted to and maintained at $\mathrm{pH} 8$ using $\mathrm{NH}_{3}(28 \%)$ as air was bubbled through the solution while being stirred for three days. Keeping the maximum $\mathrm{pH}$ at 8 ensured that ring fission of the indolquinone moieties are kept to a minimum ensuring a biomimetic material. ${ }^{34}$ Afterwards, 
the solution was brought to $\mathrm{pH} 2$ using $\mathrm{HCl}$ (32\%) to precipitate the pigment. The solution was then filtered and washed multiple times with deionised water and dried.

Cu-melanin powder was made from the melanin powder via suspension in an aqueous copper solution $\left(\mathrm{Cu}\left(\mathrm{ClO}_{4}\right)_{2}\right.$, Sigma-Aldrich) for three days. Different $\mathrm{Cu}(\mathrm{II})$-loaded melanin samples were made with different initial $\mathrm{Cu}(\mathrm{II})$ concentration loadings, which were $0.05,0.1,0.25,0.5$ \& $1.0 \mathrm{mmol}$ of $\mathrm{Cu}(\mathrm{II})$ per gram of melanin. The suspensions were all filtered and washed three times with deionised water and dried. Pellets were pressed from the powders into $13 \mathrm{~mm}$ diameter pellets using a hydraulic press, $1 \mathrm{~mm}$ thick, under 10 tons of pressure for $5 \mathrm{~min}$.

Melanin and Cu-melanin thin films: Thin film fabrication of neat melanin films utilised previously published methods. ${ }^{35}$ In brief, 0.7 g melanin powder was dissolved in water $(5 \mathrm{ml})$ and $\mathrm{NH}_{3}(10 \mathrm{ml})$. The solution was stirred for $1 \mathrm{~h}$ and ultrasonicated for $1 \mathrm{~h}$. The solution was then filtered (0.45 $\mu \mathrm{m}$ PVDF/L filter (Simplepure)) and spin coated (G3P Spincoater, Specialty Coating Systems, $5 \mathrm{~s}$ at $500 \mathrm{rpm}$ and then $60 \mathrm{~s}$ at $1500 \mathrm{rpm}$ ) onto pre-treated glass substrates. The glass substrates were cleaned with Alcanox, deionised water, ultra-sonicated in acetone (5 $\mathrm{min}$ ), rinsed with deionised water, ultra-sonicated in 2-propanol (5 min) and dried under a flow of nitrogen. Substrates were then treated with UV-Ozone (MB UV-O 3 , MBraun): 5 min of $\mathrm{O}_{2}$ purging followed by 20 min UV exposure and 5 min of $\mathrm{N}_{2}$ purging.

$\mathrm{Cu}$-loaded films were produced by using the above thin film methodology with aqueous copper solutions. The above neat melanin solution $(2 \mathrm{ml})$ was used as a stock solution. $8 \mathrm{mg}$ of $\mathrm{CuCl}_{2} \cdot 2 \mathrm{H}_{2} \mathrm{O}$ (Sigma-Aldrich) was added to fully dissolve the melanin solution before the filtering stage for slide deposition. The solution was stirred and aliquots at $1 \mathrm{~min}, 2 \mathrm{~min}$ and 5 min after the $\mathrm{CuCl}_{2} \cdot 2 \mathrm{H}_{2} \mathrm{O}$ addition were used to spin-coat onto glass. The melanin solution with the $\mathrm{Cu}$ salt added was observed to become thicker with stirring time, and after 5 min a paste resulted that could not be filtered and used. Films made with longer stirring times (e.g. 5 
min after addition) were relatively brittle. The aliquots were filtered through a $0.45 \mu \mathrm{m}$ PVDF/L filter (Simplepure). Spin-coating onto the substrates was done at $1500 \mathrm{rpm}$ for $60 \mathrm{~s}$.

Conductivity measurements on pressed powder pellets: Conductivity measurements were performed using previously published methodologies. ${ }^{14}$ Pellets were contacted using gold evaporation $(100 \mathrm{~nm})$ and silver paste. The samples were then placed in a vacuum-capable aluminium chamber with electrical feedthroughs. A Keithley 2450 source-meter unit took current-voltage (IV) (-5V to $5 \mathrm{~V})$ measurements in a four-contact configuration. The water vapour pressure in the chamber was delivered to the sample via a line attached with a BOCEdwards pressure gauge (GK series, 0-50 mbar) and a degassed, deionised water vial. In situ vapour control was achieved by evacuating the chamber, obtaining IV data and then increasing the vapour pressure in 3 mbar incremental steps. 1 hour was given for equilibrium in line with the known kinetics of melanin pellet hydration ${ }^{33}$ before IV measurements were taken. Uncertainties were determined by propagation using two times the standard error (SE). Cumelanin pellets of $0.5 \mathrm{mmol} \mathrm{g}^{-1}$ loading were also tested.

Conductivity measurements on thin films: Glass substrates were prepared as described above. $3 \mathrm{~nm}$ of chromium was evaporated thermally through a mask to be used as an adhesion layer for the next layer of gold. The gold was deposited to $50 \mathrm{~nm}$. The contact geometry was a 2point interdigitated set with a length of $100 \mu \mathrm{m}$ and width of $11.2 \mathrm{~mm}$. The melanin was then spin-coated on top. Current-voltage IVs (forward and backward sweep from -1 to $1 \mathrm{~V}, 60 \mathrm{~s}$ hold time, 1 point per sec) for the conductivity were obtained by an Agilent Semiconductor Device Analyzer B1500A. Dry thicknesses were obtained using a profilometer (DEKTAK $\mathrm{XT}$ ). The hydration procedure was performed as for the pressed powder samples. Multiple samples were measured, and the uncertainties determined to 2SE. 
OECT fabrication: Samples were fabricated and characterised as previously published ${ }^{23}$ with the exception that $\mathrm{Cu}$-melanin thin films were used as the solid-state gate electrolyte. In brief, glass substrates with contacts were fabricated as above. PEDOT:PSS (Clevios PH 1000, Heraeus Holding $\mathrm{GmbH}$ ) films were spin-coated on the substrates for $5 \mathrm{~s}$ at $500 \mathrm{rpm}$ followed by $60 \mathrm{~s}$ at $1500 \mathrm{rpm}$ and annealed for $15 \mathrm{~min}$ at $140^{\circ} \mathrm{C}$. Hexamethyldisilazane (Sigma-Aldrich) was spin-coated on top of the PEDOT:PSS at $800 \mathrm{rpm}$ for $60 \mathrm{~s}$ followed by 15 min annealing at $110^{\circ} \mathrm{C}$. The $\mathrm{Cu}$-melanin solution used was stirred for $2 \mathrm{~min}$, as it was found to be the optimal time in terms of processing. A $0.45 \mu \mathrm{m}$ hydrophobic filter was used to filter the solution before spin-coating the solution at $1500 \mathrm{rpm}$ for $60 \mathrm{~s}$. A $30 \mathrm{~nm}$ Au top gate contact was thermally evaporated. All thicknesses were measured using a Dektak XT.

OECT characterisation: The electrical characteristics of the Cu-melanin OECT devices were measured using a probe-station and an Agilent Semiconductor Device Analyser (B1500A). Transfer curves were measured with source-drain voltage, $\mathrm{V}_{\mathrm{d}}$, at $100 \mathrm{mV}$ and gate voltage, $\mathrm{V}_{\mathrm{g}}$, sweeping from $-1 \mathrm{~V}$ to $1 \mathrm{~V}$ and then back to $-1 \mathrm{~V}, 60 \mathrm{~s}$ hold time and $1 \mathrm{~s}$ delay time. All transfer and output curves were measured slowly enough to allow steady state to be reached. Output data is provided in the Supplementary Information (Figure S8). All devices (9 total) were analysed under the same laboratory conditions as previously published work. ${ }^{23}$ Data analysis was based upon the forward scan since this is the ion injecting stage. Data sets were weighted by the reciprocal of the difference between measured and mean transconductance peak voltages and analysed to 2SE.

XPS: XPS measurements were obtained using a Kratos Axis Ultra XPS Surface Analysis System with a monochromatic Al Ka X-ray source. Survey and high-resolution scans were taken at 120 and $20 \mathrm{eV}$ pass energies, respectively. The XPS spectra were analysed using CASA XPS software. Atomic ratios for $\mathrm{C}, \mathrm{N}, \mathrm{Cu}$ and $\mathrm{O}$ were determined from integrating areas under the $\mathrm{C}$ 1s, $\mathrm{N}$ 1s, $\mathrm{Cu}$ 2p and $\mathrm{O}$ 1s peaks using Shirley backgrounds for the peak modelling. 
Hydration Dependent CW EPR: Continuous Wave (CW) measurements were performed similarly to previously published work. ${ }^{17}{ }^{36}$ In brief, X-band CW EPR spectra were taken with a Bruker Elexsys E500 spectrometer with a SuperX microwave bridge, and an EN 4118X-MD4 optical resonator with a quartz liquid nitrogen insert. A thermocouple was attached to the quartz insert that was connected to a Eurotherm 4131VT variable temperature controller to monitor the sample during the experiment $\left(20.8 \pm 0.1^{\circ} \mathrm{C}\right)$. The magnetic field and microwave frequencies were calibrated with a Bruker Teslameter (ER036M) and Bruker frequency counter (E500), respectively. The magnetic field was swept between 240 and $380 \mathrm{mT}$, and a modulation frequency of $100 \mathrm{kHz}$, modulation amplitude of $0.1 \mathrm{mT}$ and a microwave power of $20 \mathrm{~mW}$ was used. In situ hydration control was obtained by a bespoke vacuum line attached to the EPR tube with the same attachments as for the conductivity measurements. The samples measured are as described above, $0.05,0.1,0.25,0.5 \& 1.0 \mathrm{mmol}$ of $\mathrm{Cu}(\mathrm{II})$ per gram of melanin. We note that the $1.0 \mathrm{mmol} \mathrm{g}^{-1}$ samples showed significant broadening of the $\mathrm{Cu}(\mathrm{II})$ features and almost no melanin free radical signal making analysis highly uncertain, however, a data set of the spectra has been included in Figure S13 for completeness.

Hydration Dependent Photo-EPR: The hydration dependent photo CW EPR experiment was performed using previously published methodologies ${ }^{25}$ utilising the above equipment and a standard white light emitting diode (see Figure S21 for the emission spectrum). Nitrogen gas was purged through the resonator to prevent residual heating. The temperature of the sample space was monitored with a thermocouple attached to the liquid nitrogen quartz insert (22.3 \pm $0.1^{\circ} \mathrm{C}$ ). Magnetic field positions for $\mathrm{Cu}(\mathrm{II})$ ( $\perp$ signals, $\left.\mathrm{g}=2.036 ; 2.0912\right)$ and the melanin radical peak ( $g=2.004,2.005$ ) were determined by performing a CW EPR scan with the light off (Figure S14). CW EPR time scans were performed by measuring the CW EPR signal intensity continuously at a fixed g value using a field frequency lock method for $167 \mathrm{~s}$. The first $50 \mathrm{~s}$ of the time scan was measured with the light off, then 50s for light on and then off for the 
remainder of the scan. The CW EPR time scans were measured at $200 \mathrm{~mW}$ and a $0.0754 \mathrm{mT}$ modulation amplitude. The CW EPR time scans were baseline corrected and filtered with a moving average filter using five neighboring data points. The signal intensities of the time scans taken at the maximum negative $\mathrm{Cu}$ (II) $\perp$ signal were multiplied by -1 /modulus taken to show the absolute signal change during illumination.

\section{Results and Discussion}

\subsection{Melanin}

Melanin is a polyindolequinone system polymerised from two monomers, dihydroxyindole (DHI) and dihydroxyindole carboxylic acid (DHICA) and their various redox states (Figure 1A). ${ }^{13}$ These monomers, under oxidative conditions, form a heterogeneous, stacked oligomer system with characteristic properties such as a broadband UV-Vis absorbance and a stable free radical signal observed clearly via EPR. ${ }^{13}$ Melanin is thus a bio-macromolecular system with physico-chemical properties that are strongly influenced by disorder at several levels of structure.

The solid state electrical conductivity of melanin is related to its underlying redox chemistry, ${ }^{15}$ notably the so-called comproportionation reaction (Figure 1B), which creates hydronium ions and hence a free carrier population of protons. The solid state conductivity can be varied as a function of the hydration state of the polymer ${ }^{14} 153738$ or temperature. ${ }^{19}{ }^{37}$ In the former case, it appears that the proton conductivity dominates with negligible electronic conductivity even though charged free radicals are created in the redox process. ${ }^{18}$ When probing the temperature dependence, additional care must be taken to ensure that the water content of the polymer does not change as the temperature is varied, ${ }^{19}$ which is difficult to achieve. As a result, most recent work has focused on hydration-dependent studies ${ }^{14} 1516373839$ which have also delivered the most mechanistic insight. 


\subsection{Melanin “Doping” by Cu(II)}

In order to probe basic material effects, we prepared $\mathrm{Cu}(\mathrm{II})$ doped melanin (Cu-melanin) by suspending pre-synthesised melanin powder (see Experimental Section) in a solution of a $\mathrm{Cu}(\mathrm{II})$ salt for three days. This allowed the $\mathrm{Cu}(\mathrm{II})$ to be incorporated into the melanin polymer via chelation in large quantities to the hydroquinone/quinone moieties. ${ }^{27} 283031$ Figure 2A shows conductivity 'isotherms' for neutral $\mathrm{pH}$, pressed powder samples of Cu-melanin as a function of hydration (water content per gram of sample) with the aim being that additional water should perturb the comproportionation reaction (as depicted in Figure 1B). We note that neutral $\mathrm{pH}$ allows for clearly observing the perturbation due to the comproportionation reaction. ${ }^{15}{ }^{17}$ Such isotherms were obtained via a now standard DC-electrical measurement protocol involving the careful measurement of equilibrated current-voltage curves using symmetric gold contacts on pressed powder pellets in a pseudo Van der Pauw configuration. It is important to note that absolute water content ( $x$-axis data) is derived from accurate analysis of equilibrium weight gained as a function of relative water pressure, i.e. detailed knowledge of the so-called adsorption isothermal behavior (see Supplementary Methods). ${ }^{14} 151733$ The conductivity (y-axis) is normalised to the dry-state conductivity to allow qualitative comparison of the Cu-melanin data to previously published ${ }^{17}$ standard (undoped) synthetic melanin results. The Cu-melanin shows the same qualitative hydration-dependent conductivity behaviour as the undoped material, i.e. a slow increase at low water contents ('dry state') and then a steep increase for higher hydrations. This qualitative behaviour is a signature of the underlying comproportionation mechanism (Figure 1B). ${ }^{15} 17$ 


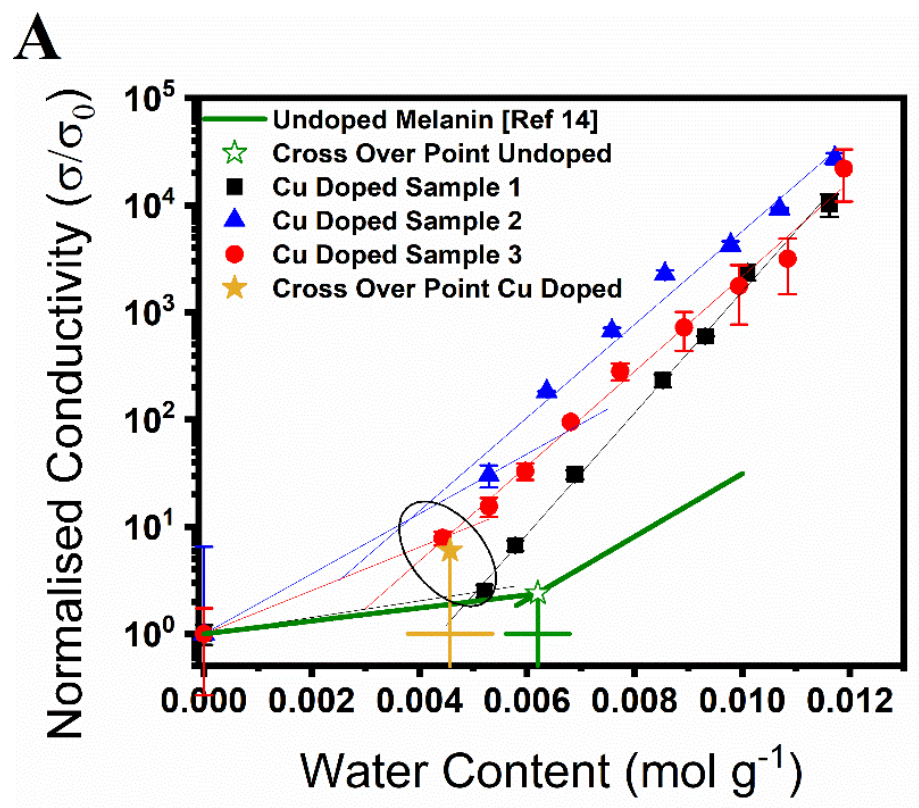

B

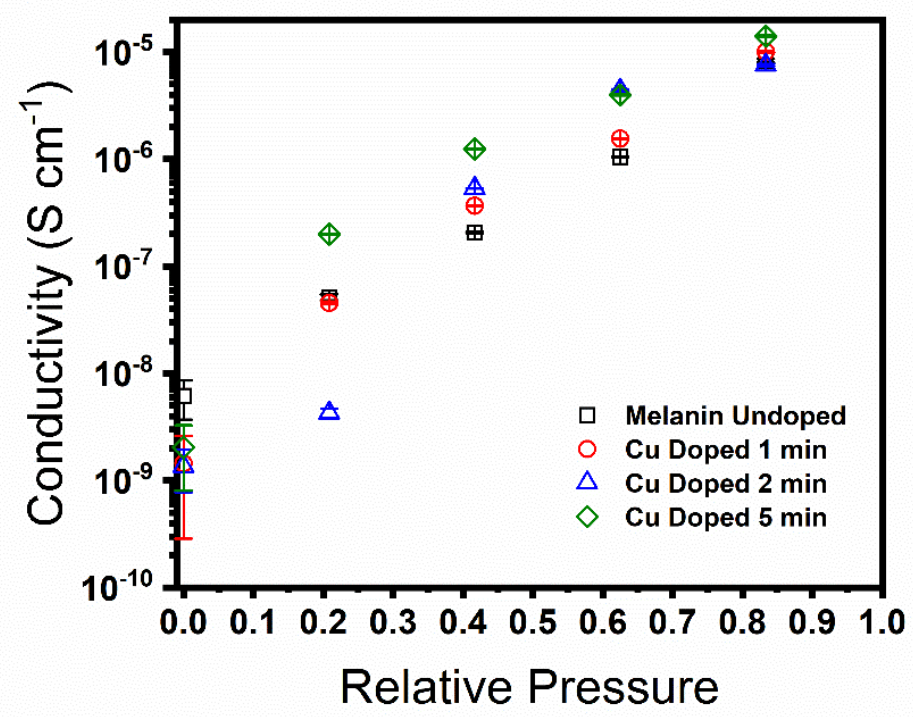

Figure 2. A) Conductivity of Cu-melanin pressed powder samples ( $\mathrm{Cu}(\mathrm{II})$ initial loading $\left.0.5 \mathrm{mmol} \mathrm{g}^{-1}\right)$ vs. water content. Absolute water content was determined from the adsorption isotherm (Figure S1). Data points are for Cu-melanin and the solid green line is reproduced from previously published data on undoped melanin. ${ }^{17}$ The stars indicate the average point of inflection between low/high hydration regimes. Horizontal bars (gold \& green) indicate the uncertainty in the inflection point ( $\left.4.57 \pm 0.79 \mathrm{mmol} \mathrm{g}^{-1} \& 6.2 \pm 0.6 \mathrm{mmol} \mathrm{g}^{-1}{ }^{17}\right)$. B) The conductivity of melanin thin films as a function of water relative pressure (i.e. relative humidity). Currently, no adsorption isotherms have been reported for thin films vs. $\mathrm{H}_{2} \mathrm{O}$ relative pressure. Conductivity values account for swelling effects which have previously been determined from Neutron Reflectometry. ${ }^{40}$ Uncertainties were determined to 2SE based upon multiple samples. Cu-concentrations are qualified by treatment times (see Figure 4 \& 5 for XRay Photoelectron Spectroscopy (XPS) elemental analysis). 
We note the following clear differences in the respective conductivity isotherms: firstly, the so-called 'cross-over point' (the point of change in gradient between the low to high hydration regimes) is shifted to drier values for Cu-melanin (stars in Figure 2A); this shift is significant relative to the 2SE error boundary. Secondly, the gradients in the wet regime are significantly higher in all Cu-melanin samples relative to the undoped material, i.e. the doped material has enhanced conductivity per mole of absorbed water relative to the undoped sample (note the log scale).

Whilst neutral $\mathrm{pH}$ pressed powder pellet samples provide a useful platform to establish the relative conductivity behavioural trends, the method of preparation (i.e. pressing the pellet), can lead to a large dry state conductivity uncertainty. ${ }^{14} 193741$ In contrast, other work on thin films of melanin have shown a morphology that produces consistent results. ${ }^{38}$ We therefore utilised thin films of Cu-melanin in conjunction with the above hydration procedure to confirm the inferences made using pressed powder pellets. Thin melanin films were spin coated from a concentrated solution of the polymer in $\mathrm{NH}_{3}$, onto pre-treated glass with a bottom-contacted set of gold interdigitated electrodes. Cu-melanin thin films were made by using the same base solution, but with a $\mathrm{Cu}(\mathrm{II})$ salt added to it with aliquots spin coated at separate times after the salt addition (1 $\mathrm{min}, 2 \mathrm{~min} \& 5 \mathrm{~min}$ ). The aforementioned times indicate the time of spin coating the film after the addition of the Cu salt to the stock solution. As can be seen in a later figure (Figure 4B), the increasing spin delay time corresponds to increasing $\mathrm{Cu}$ content.

The film conductivity results are shown in Figure $\mathbf{2 B}$. We note that the $x$-axis is in terms of relative pressure (RP) of water vapor in the surrounding measurement atmosphere. For completeness, other characterisation data for the films is presented in the Supplementary Information including UV-Vis absorbance (Figure S2 \& S3), Atomic Force Microscopy (Figure S4 - S7) and X-ray Photoelectron Spectroscopy (XPS) elemental analysis (Figure 4 \& 5). 
These device quality films allow for quantitative and qualitative comparisons between the doped and undoped materials and as such, the $y$-axis is not normalised in Figure 2B. We first note that the undoped melanin has a higher dry-state conductivity than the Cu-melanin, which is consistent with a previously published study. ${ }^{41}$ The temptation is to suggest that the melanin free radical is the residual electronic charge carrier under dry conditions, but we discount this line of reasoning since the film conductivities of all the variously doped samples in Figure 2B are the same within error. Furthermore, EPR spectra of Cu-melanin dried under vacuum in Figure 6A shows the melanin free radical concentration decreases exponentially with increasing $\mathrm{Cu}(\mathrm{II})$ concentration (the EPR results will be discussed in more detail later). However, returning to the overall trend of the datasets, it shows that on average across the hydration range that the films with higher Cu content (i.e. those with longer spin delay times) have a higher conductivity to those with less or no $\mathrm{Cu}$ content. Since the dominant carrier in melanin thin films is the proton, ${ }^{18}$ we thus infer that the proton concentration is enhanced with Cu-doping per mole of water absorbed.

The above conclusion can be further validated using a solid-state organic electrochemical transistor (ss-OECT) platform to determine the relative proton concentration in a proton-gated experiment. ${ }^{23}$ Furthermore, the fabrication of such a device provides the proof-of-concept for proton-to-electron transducing applications utilising $\mathrm{Cu}$-melanin (a relatively inexpensive and scalable material). The OECT operating mechanism is explained in detail elsewhere. ${ }^{42}$ But in brief, a semiconducting polymer such as PEDOT:PSS (polyethylene-dioxythiophene: polystyrenesulfonate) is utilised as the electronic transistor channel material (Figure 3A). Usually, an electrolyte (in our case, melanin) is used to electrochemically gate the channel driving ions (hydronium for melanin) into the PEDOT:PSS doping or de-doping the polymer backbone (see redox reaction in Figure 3Ai)) and modulating its conductivity to provide transistor behaviour. A positive gate voltage on the electrolyte, de-dopes the PEDOT:PSS 
backbone and turns the transistor off. In short, positive charge (e.g. protons) modulate OECT behaviour.

We previously published the first all solid-state manifestation of such a device using conventional melanin in a soft solid-state electrolyte capacity as a gate-source of protons. ${ }^{23}$ In this study we critically showed that the transistor characteristics could be enhanced by increasing the proton concentration of the melanin gate via hydration, namely:

a) The ON/OFF ratio increased with increasing proton concentration. The ON/OFF ratio is driven by the "off” side decrease. This occurs because the more positive ions are able to be pushed into the channel, the more de-doping can occur in the channel.

b) The transconductance (the derivative of the transfer curve) was increased (i.e. the transistor was more sensitive to gating). Essentially this is due to the same underlying reason as for the ON/OFF ratio above, since a greater ON/OFF ratio over the same voltage window will lead to a greater peak in the derivative.

c) The transconductance peak was shifted to lower voltages (i.e. the transistor turned off at lower voltages). The presence of more ions means less voltage is required to switch the channel off.

Essentially, increases in proton concentration will lead to enhanced device characteristics as per points a) to c) above. Therefore, comparing a Cu-melanin based OECT to a melanin based OECT should show a difference in performance, with the Cu-melanin possessing the enhanced behaviour if all other parameters are kept the same (Figure 3Aii).

The Cu-melanin OECT characterisation with a nominally identical undoped melanin OECT is shown in Figure 3B \& C. The transistor characteristics are summarised in the corresponding table (Table 1). 

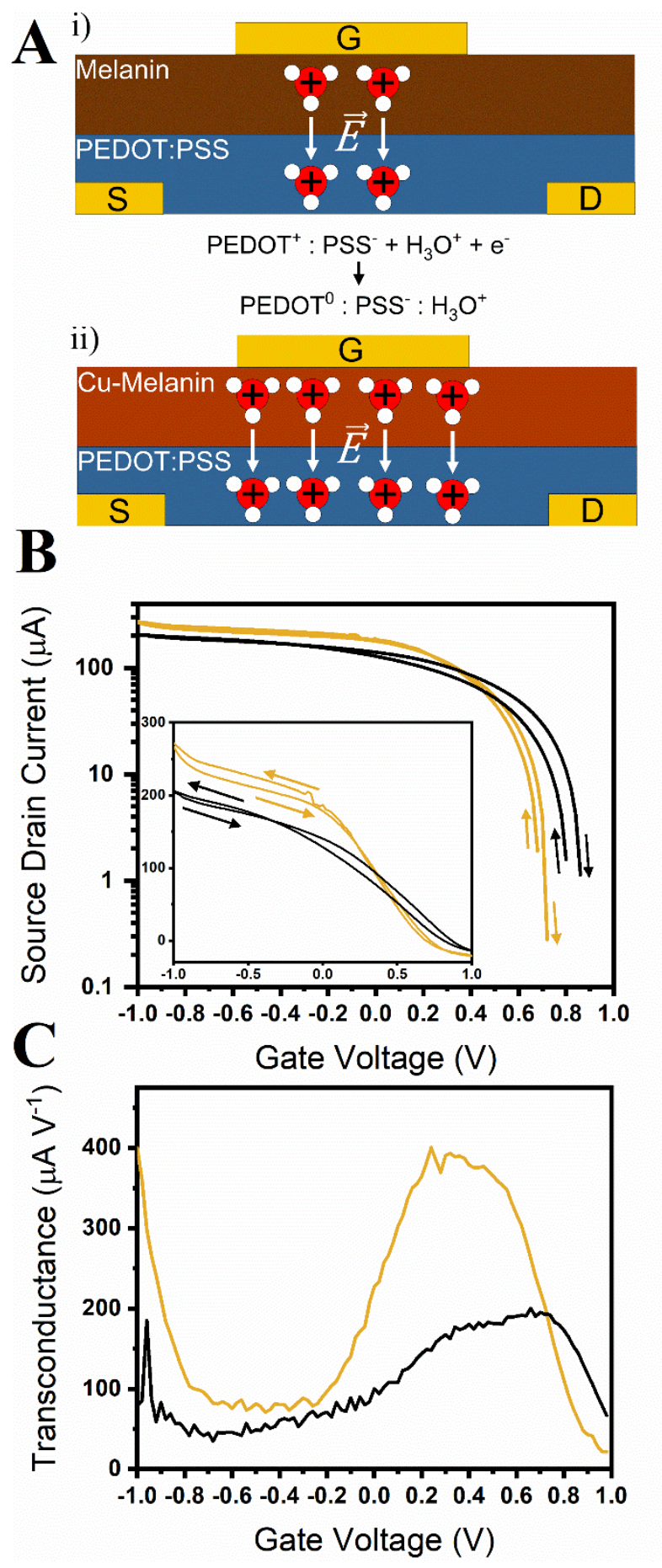

Figure 3. A) Schematic of the operation of an OECT. Under the action of an electric field due to the gate (G), positive ions (hydronium) are moved from the solid state electrolyte (i) melanin or ii) Cu-melanin) into the active channel (PEDOT:PSS), where the ions charge compensate the PEDOT:PSS backbone. This charge compensation eliminates the holes from the PEDOT (PEDOT ${ }^{+} \rightarrow$ PEDOT $^{0}$, see ii) for full redox reaction) and thus dedopes/lowers the current in the channel between the source (S) and the drain (D). Shown between i) and ii) is the expected conceptual difference between the melanin and $\mathrm{Cu}$-melanin, i.e. higher proton concentration in $\mathrm{Cu}-$ melanin. B) Example transfer characteristics for the OECT devices. Inset is the same data but on a linear-linear 
scale. Note that at high positive voltages the currents become slightly negative, hence the absence of data on the log-linear plot above $0.8 \mathrm{~V}$. The scans were obtained by scanning from left to right. Arrows indicate the forward and backward scans. C) Example transconductance characteristic for the OECTs devices (forward scan). Cumelanin based OECTs (gold) and previously published melanin based OECTs (black). ${ }^{23}$

Table 1. Values of the different OECT devices based upon melanin and doped melanin. All uncertainties to 2SE.

\begin{tabular}{llll}
\hline Type & ON/OFF Ratio & $\begin{array}{l}\text { Transconductance Peak } \\
\left(\mu \mathrm{AV}^{-1}\right)\end{array}$ & $\begin{array}{c}\text { Transconductance } \\
\text { Voltage (V) }\end{array}$ \\
\hline Melanin OECT & & \\
& $360 \pm 260$ & $150 \pm 20$ & $0.52 \pm 0.03$ \\
Cu-melanin OECT & $640 \pm 340$ & $300 \pm 60$ & $0.38 \pm 0.05$ \\
\hline
\end{tabular}

${ }^{\mathrm{i}}$ Previous data sets (11 devices) were analysed as per Cu-melanin OECT analysis described in the Experimental Section.

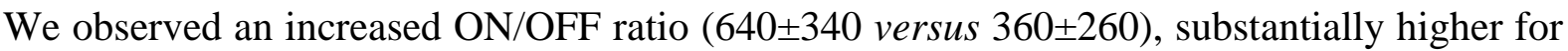
Cu-melanin OECTs but not enough to overcome the bounds of uncertainty. The most likely reason for the wide bounds is that small negative currents are measured in the OFF state for the devices, making a true estimation of an ON/OFF ratio difficult. However, an alternative to the ON/OFF ratio, which is not affected by the above problem are the peak values of the transconductances $\left(150 \pm 20\right.$ versus $\left.300 \pm 60 \mu \mathrm{AV}^{-1}\right)$, which clearly shows a greater value for the Cu-melanin OECTs. This strongly supports the presence of a relatively greater number of protons in Cu-melanin as compared to neat melanin (i.e. point b) above). Further support, as to point c) above, is seen via a significant shift of the transconductance peak to a lower voltage operation: $0.38 \pm 0.05$ versus $0.52 \pm 0.03 \mathrm{~V}$ for $\mathrm{Cu}$-melanin OECTs and neat melanin OECTs respectively. Hence, we conclude that our inference of an increase in proton concentration in the Cu-melanin film and pellet conductivity measurements (Figure 2) is strongly supported by 
the OECT results. Furthermore, an inspection of the leakage current (Figure S8) between neat and Cu-melanin OECTs show an increase in the current for Cu-melanin OECTs relative to the neat devices. This is also consistent with more mobile charges, i.e. protons, being present in the Cu-melanin devices.

In the next section we will investigate the chemistry of the Cu-melanin interactions and a potential mechanism that explains the above aided by EPR results.

\subsection{Copper Chemistry}

Our first step in understanding the doping mechanism was to confirm the oxidation state of $\mathrm{Cu}$ in Cu-melanin. This is important since redox chemistry is responsible for the proton conductive behaviour (Figure 1B), and we thus anticipate that the oxidation state will play an important role. Given melanin's notorious insolubility, ${ }^{13}$ determining elemental composition is usually facilitated using XPS, which may also provide insight to the aforementioned redox state of the

Cu. ${ }^{15}{ }^{40}$ As demonstrated in an example XPS spectrum (Cu-melanin film, 5 min after $\mathrm{Cu}$ addition) and associated analysis (see Figure 4A), the C: O: $\mathrm{N}$ atomic ratios are commensurate with a standard melanin polymer. Furthermore, the presence of the $\mathrm{Cu}$ peak indicates that the copper was successfully incorporated into the material. For Cu-melanin films specifically, which are spin coated after some time after $\mathrm{CuCl}_{2}$ addition to the stock solution (e.g. 1 min, 2 $\min \& 5 \mathrm{~min}$ ), we show that the $\mathrm{Cu}$ content increases the longer the stock solution is stirred (see Figure 4B). The $\mathrm{Cl}$ content concomitantly rises as expected due to the use of $\mathrm{CuCl}_{2}$ as the additive salt. Using the atomic percentages as indicated in Figure 4B, one can obtain for the 5 min film a Cu content of $\sim 0.54 \mathrm{mmol} \mathrm{g}^{-1}$. For $1 \mathrm{~min}$ and 2 min similarly $\sim 0.19 \mathrm{mmol} \mathrm{g}^{-1} \&$ $\sim 0.43 \mathrm{mmol} \mathrm{g}^{-1}$ respectively. However, we note that these estimations are only indicative. The atomic percentages used, for example, are determined by excluding hydrogen content, since XPS cannot determine the hydrogen content samples. We also did not utilise the $\mathrm{Cu} 2 \mathrm{p}_{1 / 2}$ peak 
( $953 \mathrm{eV}$ ), since the baseline could not be determined due to the skewed background from the large higher order O step present $\sim 1000 \mathrm{eV}$.

Looking to more specific detail of the oxidative states, we note that generally, $\mathrm{Cu}(\mathrm{II})$ has been observed in CW EPR spectra of Cu-melanin in the past and supposed redox reactions have been discounted in favour of magnetic screening effects. ${ }^{13}{ }^{29}$ Hence, the presence of a significant XPS shoulder corresponding to $\mathrm{Cu}(\mathrm{I})$ was surprising. This can be seen in an example high resolution XPS scan in Figure 5. This data and indicates that $\mathrm{Cu}(\mathrm{II})$ can undergo a redox reaction with melanin in significant amounts.

A

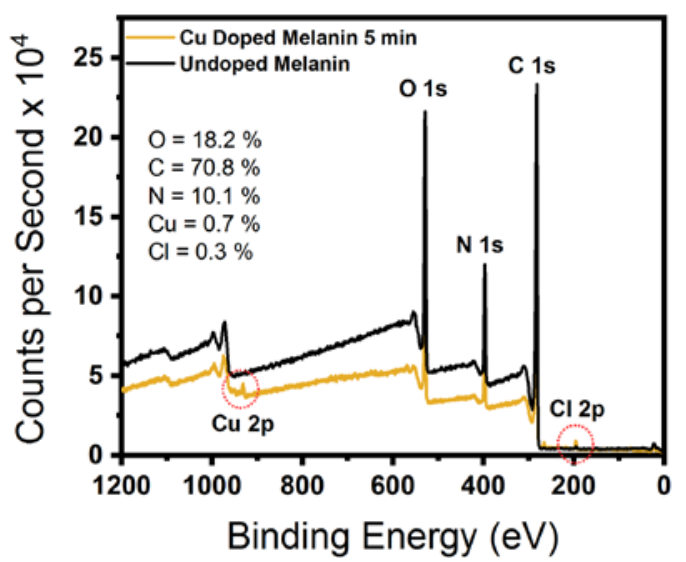

B

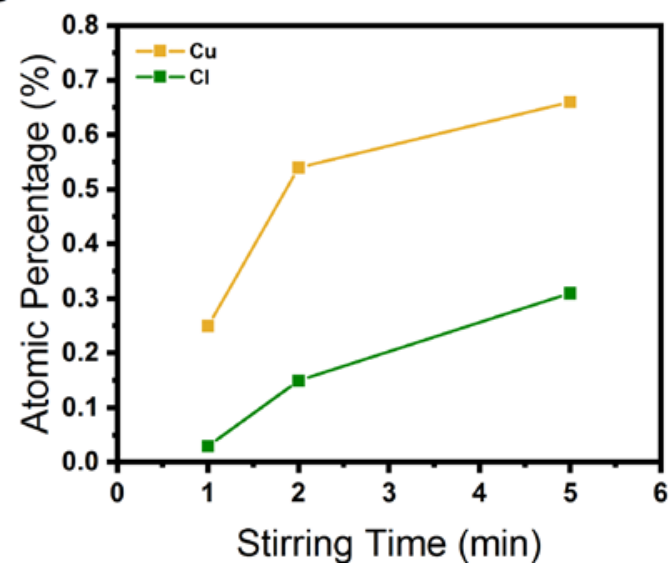

Figure 4. A) Example XPS scans obtained on 1) a Cu-melanin film on a glass substrate spin coated 5 min after $\mathrm{Cu}$ addition (gold), and 2) a neat melanin film on a glass substrate (black). Example XPS scans obtained on Cumelanin films on glass substrates fabricated from a solution spun cast for 5 min (gold), and melanin films on glass substrate (black). The curves are offset for clarity. Similar results were seen for powder Cu-melanin. The expected C:O:N ratios for melanin films are between $72 \%: 18 \%: 9 \%$ and $64 \%: 29 \%: 7 \%$ based upon the initial starting monomers. The ratios obtained experimentally indicates that $\mathrm{Cu}$-melanin are very much a melanin based material. B) Atomic percentages obtained from XPS of $\mathrm{Cu}$ and $\mathrm{Cl}$ in $\mathrm{Cu}$-melanin thin films as a function of a solution stirring time. The data clearly indicates that the $\mathrm{Cu}$ content is increasing with stirring time. The $\mathrm{Cl}$ presence is due to $\mathrm{CuCl}_{2} \cdot 2 \mathrm{H}_{2} \mathrm{O}$. For a list of atomic percentage, see Table $\mathbf{S} 2$. 


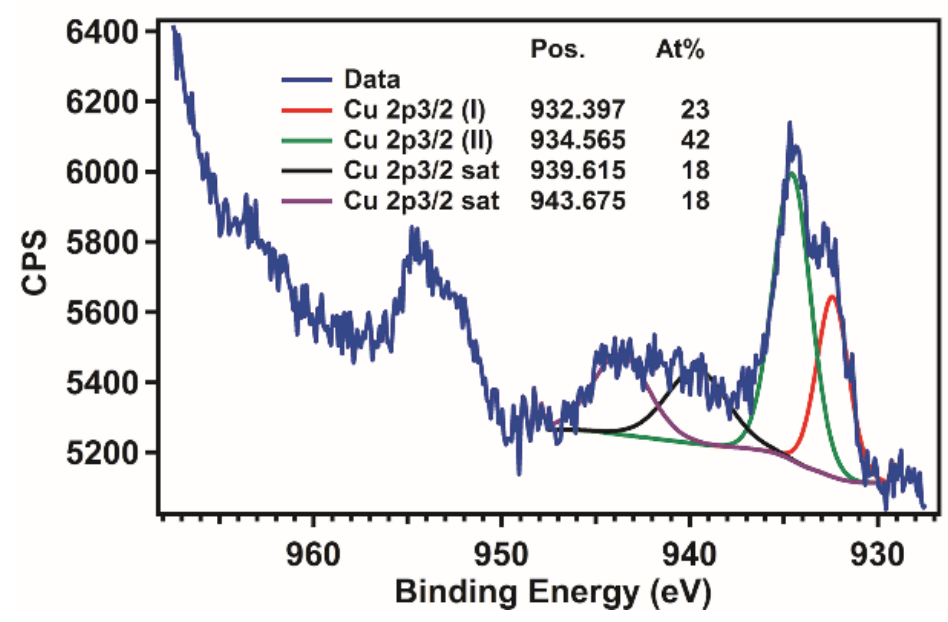

Figure 5. An example high-resolution scan of a $0.5 \mathrm{mmol} \mathrm{g}^{-1} \mathrm{Cu}-$ melanin powder. The data indicates that $\mathrm{Cu}(\mathrm{I})$ and $\mathrm{Cu}(\mathrm{II})$ are present in comparable amounts [Cu (satellite peaks also indicated)].

The satellite peaks (Figure 5) in the XPS spectra with the additional aforementioned broad oxygen peak at high binding energy makes it difficult to fully quantify the relative $\mathrm{Cu}(\mathrm{I})$ and $\mathrm{Cu}(\mathrm{II})$ content. In order to estimate the $\mathrm{Cu}(\mathrm{I}) / \mathrm{Cu}(\mathrm{II})$ content, an EPR experiment using $\mathrm{Cu}(\mathrm{II})$ glycine standards was performed (see Figure S18 - S20 and Table S3) to quantify Cu(II) in the polymer. For an initial loading of $0.5 \mathrm{mmol} \mathrm{g}^{-1} \mathrm{Cu}(\mathrm{II})$ per gram of melanin, the $\mathrm{Cu}(\mathrm{I}) / \mathrm{Cu}(\mathrm{II})$ ratio was estimated to be $\sim 3: 1$, which indicated that most of the $\mathrm{Cu}(\mathrm{II})$ was reduced to $\mathrm{Cu}(\mathrm{I})$ upon incorporation into the polymer. These observations strongly suggest that melanin, which has a well-known and persistent free radical EPR signal, ${ }^{13}$ undergoes redox chemistry upon addition of $\mathrm{Cu}(\mathrm{II})$. This new chemical analysis on $\mathrm{Cu}$-melanin also provides much needed clarity around the long-standing assumption that magnetic screening effects alone were responsible for the characteristic CW-EPR spectra observed for different concentrations of Cumelanin. ${ }^{13} 29$

\subsection{Mechanistic Proposal}

\subsubsection{Introduction to EPR Data}

With insight from the oxidation state of $\mathrm{Cu}$ from XPS, we explore the behaviour of $\mathrm{Cu}$ (II) using EPR to elucidate a potential chemical mechanism for the observations in Figures 1 - 3. Next 
we performed an in situ hydration-controlled CW EPR experiment on melanin pellet samples with various $\mathrm{Cu}(\mathrm{II})$ loadings. The main results are shown in Figure 6 (see also Figure S9 S17). In Figure 6A the relative melanin CW EPR signal is shown as a function of the initial $\mathrm{Cu}(\mathrm{II})$ loading, all obtained under vacuum to investigate the baseline behaviour of the $\mathrm{Cu}(\mathrm{II})$ loading while excluding potential hydration effects. First, we note that the data in the inset of Figure 6A is normalised to the peak-to-peak height of the $\mathrm{Cu}$ perpendicular $(\perp)$ peak, i.e. $\mathrm{Cu}(\mathrm{II})$. This turns out to be a reasonable method to compare the relative melanin signal behaviour as a function of total Cu content since i) changes in the peak-to-peak height in EPR spectra are proportional to changes in free radical concentration, and ii) at low $\mathrm{Cu}$ loadings (e.g. $0.05 \mathrm{mmol} \mathrm{g}^{-1}$ ) most of the $\mathrm{Cu}$ is in the $\mathrm{Cu}(\mathrm{II})$ oxidation state. ${ }^{32}$ The inset of Figure $6 \mathbf{A}$ shows the melanin free radical concentration decreases exponentially as a function of initial $\mathrm{Cu}(\mathrm{II})$ loading. Furthermore, as the $\mathrm{Cu}(\mathrm{II})$ content is increased, the $\mathrm{Cu}(\mathrm{II})$ signal undergoes dipolar broadening at relatively high $\mathrm{Cu}(\mathrm{II})$ loading $\left(0.5 \mathrm{mmol} \mathrm{g}^{-1}\right)$, indicating that the $\mathrm{Cu}$ (II) centers are closer together and being affected by other nearby $\mathrm{Cu}(\mathrm{II})$ centers (see for example reference $^{43}$ ). These results are consistent with CW EPR spectra of Cu-melanin solutions in the literature where it has been speculated a possible reduction of $\mathrm{Cu}(\mathrm{II})$ to $\mathrm{Cu}(\mathrm{I})$ could be occurring, but remained unconfirmed due to $\mathrm{Cu}(\mathrm{I})$ being diamagnetic (which cannot be directly detected with EPR). ${ }^{1329}$ 
A

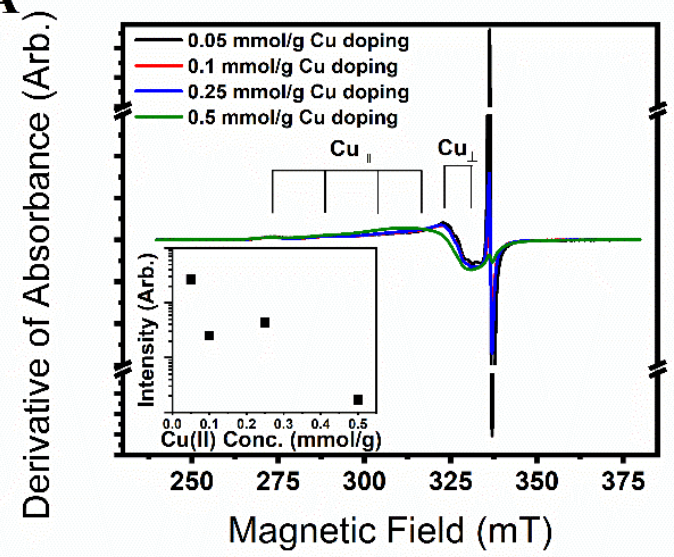

C

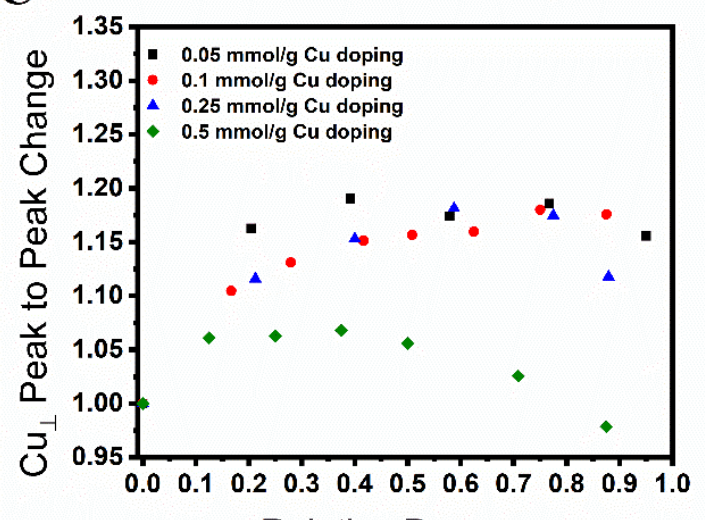

B

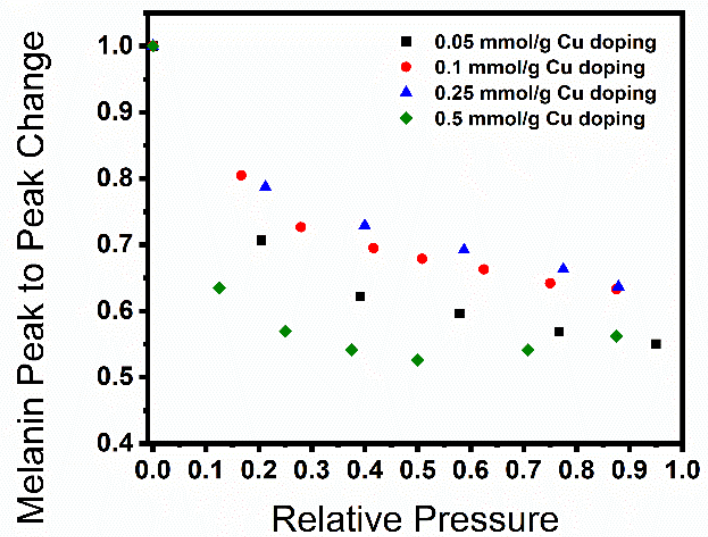

D

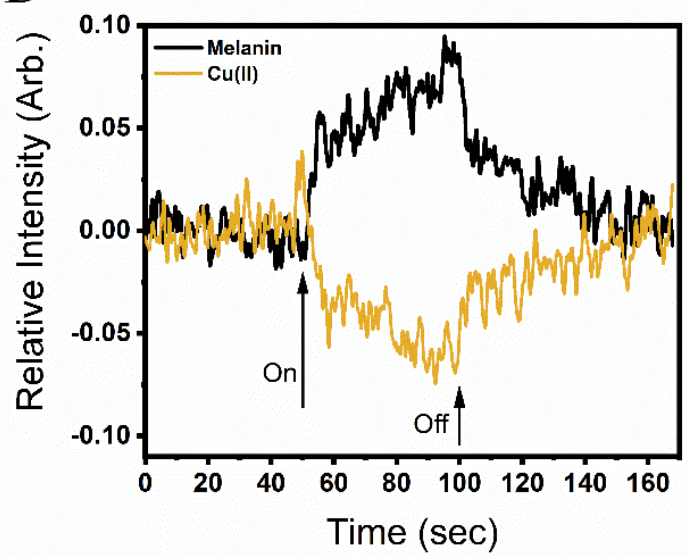

Figure 6. A) CW EPR spectra of Cu-melanin pellets dried under vacuum. The data shows \| Cu(II) signals at low magnetic field and the $\perp$ signal at $\sim 330 \mathrm{mT}$. Note that $\mathrm{Cu}(\mathrm{I})$ is EPR inactive. At $\sim 337.5 \mathrm{mT}$ the well-known melanin peak is evident. The data was obtained at $20 \mathrm{~mW}$ and all curves have been normalised to the peak-topeak height of the $\perp$ Cu EPR component. The inset indicates the peak-to-peak height of the melanin EPR signal. B) The peak-to-peak intensity of the melanin feature as a function of hydration (relative to the dry data), which is recorded as the relative pressure (RP) of the water vapour for variously doped Cu-melanin samples. C) The peakto-peak intensity of the $\perp \mathrm{Cu}$ (II) component as a function of hydration relative to the dry data set for variously doped Cu-melanin samples. D) Photo CW EPR time scan of the melanin peak and the dip of the $\perp$ Cu(II) peak for $0.25 \mathrm{mmol} \mathrm{g}^{-1} \mathrm{Cu}$-melanin, taken at 0.5 relative pressure. Data obtained at $200 \mathrm{~mW}$ (see text for details). ${ }^{25} \mathrm{The}$ positions of light on/off are indicated. Note that the modulus has been applied and hence one is observing the absolute intensity change relative to the peak before illumination.

\subsubsection{Hydration Dependent Signal and its Relation to the Supposed Carbon Centered Radical}

Melanin contains multiple radical species, which makes EPR analysis of such spectra more complex. ${ }^{17} 2536444546$ In the solid-state, the dry melanin CW EPR signal is dominated by an 
apparent carbon-centered radical (CCR), the exact nature of which is still to be determined. ${ }^{36}$ ${ }^{46}$ Hence, the data in Figure 6A suggests that by increasing the amount of $\mathrm{Cu}(\mathrm{II})$, the CCR is being eliminated. Furthermore, given that once incorporated, $\mathrm{Cu}(\mathrm{II}$ ) is reduced to $\mathrm{Cu}(\mathrm{I}$ ) (as discussed earlier) a very simple reaction may be inferred:

$\mathrm{Cu}(\mathrm{II})$ complex $+\mathrm{CCR}^{\bullet} \leftrightarrow \mathrm{Cu}(\mathrm{I})$ complex + Monomer

where the 'Monomer' indicates a moiety in the polymer that has been oxidised and is no longer a radical. Note that the mechanism is inferred under dry, oxygen less conditions (i.e. vacuum).

Considering the hydration-dependent effects, it has been established previously that the total solid-state organic CW EPR signal of melanin decreases linearly with increasing hydration, due to an actual decrease of the total free radical concentration. ${ }^{36}$ From an inspection of Figure 6B it is evident that for the variously $\mathrm{Cu}(\mathrm{II})$-loaded melanin polymers, the peak-to-peak height of the melanin peak decreases linearly, i.e. that the CCR concentration is also decreasing linearly. Given that the magnitude of the observed decreases is comparable to that observed in the neat polymer, ${ }^{36}$ the plausible explanation is that the same underlying mechanism is at play and as such there is a basic reaction proceeding according to:

$2 \mathrm{CCR}^{\bullet} \stackrel{\mathrm{H}_{2} \mathrm{O}}{\longleftrightarrow} 2$ Monomer

where the $\mathrm{H}_{2} \mathrm{O}$ indicates that in the presence of water the reaction favours monomer formation.

As illustrated in Figure 6C, the intensity of the perpendicular $\mathrm{Cu}(\mathrm{II})$ peak-to-peak signal generally increases with hydration. This can be explained using Equation 1 and 2 above, since water causes a decrease in the CCR (Equation 2), the chemical potential void left by the CCR is compensated by an enhanced reaction in the reverse direction (Equation 1). The net result, a consequence of Le Chatelier's principle, is the regeneration of $\mathrm{Cu}(\mathrm{II})$ complexes. The one partial exception is data for a $\mathrm{Cu}(\mathrm{II})$ loading of $0.5 \mathrm{mmol} \mathrm{g}^{-1}$, where initially the same trend is 
observed, but then at high water content the peak-to-peak intensity decreases. It should be noted that the CW EPR signal shows broadening of the $\mathrm{Cu}(\mathrm{II})$ features indicating dipolar broadening effects. This dipolar broadening is due to a complex interplay between interacting $\mathrm{Cu}(\mathrm{II})$ centers and the increasing water content. This manifests itself as an artifact of the analysis in which the signal intensity maxima is no longer strictly proportional to the concentration of $\mathrm{Cu}(\mathrm{II})$ at relatively high $\mathrm{Cu}(\mathrm{II})$ loading. Nevertheless, these EPR results from the $\mathrm{Cu}(\mathrm{II})$ loading of $0.5 \mathrm{mmol} \mathrm{g}^{-1}$ are included here to importantly highlight the challenges in examining $\mathrm{Cu}-$ melanin properties without systematically controlling $\mathrm{Cu}(\mathrm{II})$ loading and water content. Due to the sensitivity of EPR, there is inevitably also limitations on how much $\mathrm{Cu}(\mathrm{II})$ (or any metal ion) can be incorporated into an EPR sample before spin-spin interactions between paramagnetic centers become predominant and no longer negligible.

\subsubsection{Investigating the Semiquinone EPR Component Using Photo-EPR}

Thus far, our discussion has focused on the CCR component in melanin, and not the more relevant semiquinone radical, which forms an integral part of the comproportionation reaction, and which is widely believed to be central to the proton concentration and thus conductivity ${ }^{47}$. The CCR is explicitly discounted since its behaviour does not follow the conductivity ${ }^{17}{ }^{36}$ and as such cannot be linked to the proton concentration, whereas the semiquinone concentration does follow the conductivity/comproportionation reaction. ${ }^{15} 47$

The semiquinone is part of the overall organic EPR melanin signal but is only observable as a minor component relative to the CCR in solid-state. However, a recently published methodology is now available to facilitate qualitative insight into the semiquinone behaviour in the solid-state. By wetting a melanin sample, increasing the microwave power and coupling in optical light, ${ }^{25}$ a positive photo CW EPR time trace signal can be obtained which is due to semiquinone generation. As can be seen in Figure 6D (and Figure S14 \& S15), there is a 
positive photo-EPR signal, indicating that in Cu-melanin the semiquinone is definitely present as expected. However, if the equivalent photo-EPR experiment is performed on the $\mathrm{Cu}(\mathrm{II})$ signals (Figure 6D and Figure S14, S16 \& S17), there is a decrease of intensity upon illumination. This clearly indicates that the $\mathrm{Cu}(\mathrm{II})$ center is undergoing reduction and is forming $\mathrm{Cu}(\mathrm{I})$ (which is diamagnetic and EPR inactive) via the process:

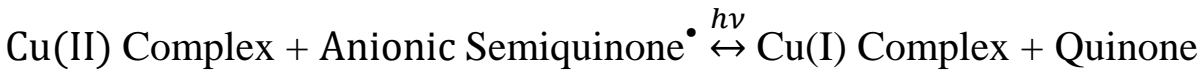

where the quinone (Figure 1A) is the product formed upon semiquinone oxidation.

\subsubsection{The Proposed Mechanism}

From the above results there are two key observations:

1. The comproportionation reaction is still active in the $\mathrm{Cu}$-melanin material as per the conductivity results - with both the proton concentration and the generation of protons per water molecule being enhanced.

2. Generating semiquinone radicals leads to $\mathrm{Cu}(\mathrm{II})$ reduction.

The simplest mechanism to explain the above observations is depicted in Figure $\mathbf{7}$ for two parallel reactions. The first is the usual comproportionation reaction (top of Figure 7). The second (bottom of Figure 7) links semiquinone radical formation with $\mathrm{Cu}(\mathrm{II})$ reduction. The key to this proposed mechanism (and the resultant population kinetics) is the interplay between the two reactions. By way of summary, as semiquinones and protons are generated upon water addition to the polymer (top reaction), an excess of semiquinones thus generated reduce nearby $\mathrm{Cu}(\mathrm{II})$ complexes. This reaction in turn generates quinone moieties that then feedback into the comproportionation reaction to generate further protons. These interconnected reactions generate the enhanced proton concentration per mole of adsorbed water at equilibrium that we observe in the electrical data in the doped samples. 


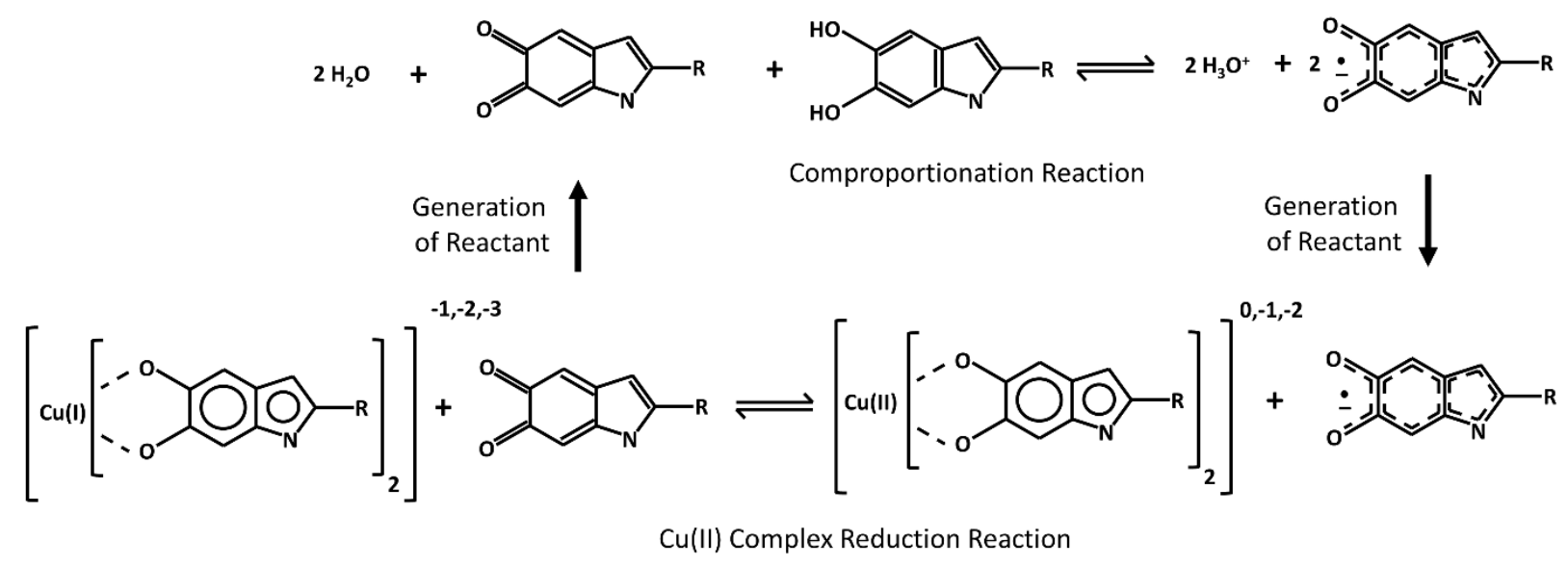

Figure 7. The proposed mechanism for enhanced proton generation in Cu-melanin. Essentially, two reactions in parallel, the comproportionation reaction (top) is depicted with a $\mathrm{Cu}(\mathrm{II})$ complex reaction with a semiquinone radical depicted (bottom) (reading from right to left). Hydroquinone and quinone moieties react with water to generate protons and semiquinones as per Figure 2B. The semiquinones generated feed into the reaction below as a reactant and reduces $\mathrm{Cu}(\mathrm{II})$. The quinones then feed back into the comproportionation reaction as a reactant to continue the cycle of proton generation, hence producing more protons for a given hydration. The cycle is controlled by the respective equilibrium constants of both reactions.

Finally, it is worth noting that the above mechanism, more broadly, may have similar physicochemical effects on the polydopamine systems, which are essentially a simpler version of melanins containing quinone and hydroquinone moieties for binding. ${ }^{48}$ Polydopamines have recently been identified as potentially useful functional materials for a range of applications. ${ }^{49}$ Furthermore, given that the $\mathrm{Cu}(\mathrm{II})$ ions form complexes with the hydroquinone moieties in melanin, any other transition metal ions that complex likewise are potential candidates for proton concentration enhancements e.g. Fe(II), Fe(III), Zn(II), Ni(II) or Co(II). ${ }^{27} 28$ Expanding the palette of biocompatible, soft solid-state ionic conductors will drive further progress in the development of coupled ion-electron devices such as artificial synapses, sensing transistor platforms and proton diodes for proton logic applications. 


\section{Conclusion}

Melanins have emerged as archetypal soft, ionic conductors and sustain proton currents over relatively large dimensions of the order of millimeters if not centimeters. We have developed a strategy that uses copper ion doping to modify the electrical properties of melanin - notably, we can enhance conductivity (carrier density) by adding copper ions using solutions of Cu(II) salts post polymer synthesis. We have studied the impact of such a process on melanin powder pellets and thin films, and confirmed the underlying observations using an all solid-state OECT platform with Cu-doped melanin as a top gate electrolyte. We have also performed detailed dark-and-photo-EPR measurements as a function of hydration and doping to measure the relative radical populations. From this analysis we propose a simple, coupled two-step reaction mechanism which invokes a competition between $\mathrm{Cu}(\mathrm{II})$ reduction and semiquinone production. In principle the same strategy should be directly translatable to relatives of the polyindolequinone family of solid-state ionic conductors e.g. polydopamine. Furthermore, the underlying mechanism indicates that a wide array of other transition metal ions should yield a similar effect, allowing more precise charge transport engineering.

\section{Conflicts of Interest}

There are no conflicts of interest to declare.

\section{Acknowledgements}

G.S. and J.R.H acknowledges the Australian Research Council for financial support in the form of a Future Fellowship (FT120100694 and FT120100421). P.M. acknowledges the Australian Research Council through its Discovery Program (DP140103653) and the Welsh European Funding Office (European Regional Development Fund) through the Sêr Cymru II Program. P.M. is a Sêr Cymru II National Research Chair. A.B.M. is a Sêr Cymru II Fellow and the results incorporated in this work have received funding from the European Union's Horizon 2020 research and innovation program under the Marie Skłodowska-Curie grant agreement No 663830. S.B.R. was funded by the University of Queensland International Scholarship. M.S. was funded by the University of Queensland International Postgraduate Research Scholarship. This work was performed in part at the Queensland node of the Australian National Fabrication Facility (ANFF-Q), a company established under the National Collaborative Research Infrastructure Strategy to provide nano- and microfabrication facilities for Australian researchers. The authors acknowledge funding from the University of Queensland (Strategic Initiative, Centre for Organic Photonics \& Electronics). The authors also acknowledge the facilities, and the scientific and technical assistance, of the Australian Microscopy \& Microanalysis Research 
Facility at the Centre for Microscopy and Microanalysis, The University of Queensland. The authors particularly want to thank Dr. Barry Wood for his guidance with the XPS measurements and analysis.

\section{References}

1. M. Bixon, B. Giese, S. Wessely, T. Langenbacher, M. Michel-Beyerle and J. Jortner, Proceedings of the National Academy of Sciences USA, 1999, 96, 11713-11716.

2. D. Porath, A. Bezryadin, S. de Vries and C. Dekker, Nature, 2000, 403, 635-638.

3. R. Endres, D. Cox and R. Singh, Review of Modern Physics, 2004, 76, 195-214.

4. A. Houlton and S. M. D. Watson, Annual Reports on the Progress of Chemistry A, 2011, 107, 21-42.

5. R. C. G. Creasy, A. B. Mostert, T. A. H. Nguyen, B. Virdis, S. Freguia and B. Laycock, Acta biomaterialia, 2018, 69, 1-30.

6. T. Miyake and M. Rolandi, Journal of Physics: Condensed Matter, 2016, 28, 023001.

7. M. Irimia-Vladu, Chemical Society Reviews, 2014, 43, 588-610.

8. P. Meredith, C. J. Bettinger, M. Irimia-Vladu, A. B. Mostert and P. E. Schwenn, Reports on Progress in Physics, 2013, 76, 034501.

9. $\quad$ N. Amdursky, E. D. Glowacki and P. Meredith, Advanced Materials, 2019, 31, 1802221.

10. M. Torculas, J. Medina, W. Xue and X. Hu, ACS Biomaterials Science and Engineering, 2016, 2, 1211-1223.

11. M. Muskovich and C. J. Bettinger, Advanced Healthcare Materials, 2012, 1, 248-266.

12. N. S. Malvankar, M. Vargas, K. P. Nevin, A. E. Franks, C. Leang, B. C. Kim, K. Inoue, T. Mester, S. F. Covalla, J. P. Johnson, V. M. Rotello, M. T. Tuominen and D. R. Lovley, Nature Nanotechnology, 2011, 6, 573-579.

13. P. Meredith and T. Sarna, Pigment Cell Research, 2006, 19, 572-594.

14. A. B. Mostert, B. J. Powell, I. R. Gentle and P. Meredith, Applied Physics Letters, 2012, 100, 093701.

15. A. B. Mostert, B. J. Powell, F. L. Pratt, G. R. Hanson, T. Sarna, I. R. Gentle and P. Meredith, Proceedings of the National Academy of Sciences USA, 2012, 109, 89438947.

16. J. Wünsche, F. Cicoira, C. F. O. Graeff and C. Santato, Journal of Materials Chemistry B, 2013, 1, 3836-3842.

17. S. B. Rienecker, A. B. Mostert, G. Schenk, G. R. Hanson and P. Meredith, Journal of Physical Chemistry B, 2015, 119, 14994-15000.

18. M. Sheliakina, A. B. Mostert and P. Meredith, Advanced Functional Materials, 2018, 28, 1805514.

19. K. A. Motovilov, V. Grinenko, M. Savinov, Z. V. Gagkaeva, L. S. Kadyrov, A. A. Pronin, Z. V. Bedran, E. S. Zhukova, A. B. Mostert and B. P. Gorshunov, RSC Advances, 2019, 9, 3857-3867.

20. P. Kumar, E. Di Mauro, S. Zhang, A. Pezzella, F. Soavi, C. Santato and F. Cicoira, Journal of Materials Chemistry C, 2016, 4, 9516-9525.

21. Y. J. Kim, W. Wu, S. Chun, J. F. Whitacre and C. J. Bettinger, Proceedings of the National Academy of Sciences USA, 2013, 110, 20912-20917.

22. M. P. da Silva, J. C. Fernandes, N. B. de Figueredo, M. Mulato and C. F. O. Graeff, AIP Advances, 2014, 4, 037120.

23. M. Sheliakina, A. B. Mostert and P. Meredith, Material Horizons, 2018, 5, 256-263.

24. K. D. Kreuer, S. J. Paddison, E. Spohr and M. Schuster, Chemical Reviews, 2004, 104, 4637-4678. 
25. A. B. Mostert, S. B. Rienecker, C. Noble, G. R. Hanson and P. Meredith, Science Advances, 2018, 4, eaaq1293.

26. S. Chio, J. S. Hyde and R. C. Sealy, Archives of Biochemistry and Biophysics, 1982, 215, 100-106.

27. C. C. Felix, J. S. Hyde, T. Sarna and R. C. Sealy, Journal of the American Chemical Society, 1978, 100, 3922-3926.

28. L. Hong and J. D. Simon, Journal of Physical Chemistry B, 2007, 111, 7938-7947.

29. T. Sarna, J. S. Hyde and H. M. Swartz, Science, 1976, 192, 1132-1134.

30. W. Froncisz, T. Sarna and J. S. Hyde, Archives of Biochemistry and Biophysics, 1980, 202, 289-303.

31. T. Sarna, W. Froncisz and J. S. Hyde, Archives of Biochemistry and Biophysics, 1980, 202, 304-313.

32. M. S. Blois, A. B. Zahlan and J. E. Maling, Biophysical Journal, 1964, 4, 471-490.

33. A. B. Mostert, K. J. P. Davy, J. L. Ruggles, B. J. Powell, I. R. Gentle and P. Meredith, Langmuir, 2010, 26, 412-416.

34. M. d'Ischia, K. Wakamatsu, A. Napolitano, S. Briganti, J. C. Garcia-Borron, D. Kovacs, P. Meredith, A. Pezzella, M. Picardo, T. Sarna, J. D. Simon and S. Ito, Pigment Cell Research, 2013, 26, 616-633.

35. J. Bothma, J. de Boor, U. Divakar, P. Schwenn and P. Meredith, Advanced Materials, 2008, 20, 3539-3542.

36. A. B. Mostert, G. R. Hanson, T. Sarna, I. R. Gentle, B. J. Powell and P. Meredith, Journal of Physical Chemistry B, 2013, 117, 4965-4972.

37. M. Jastrzebska, H. Isotalo, J. Paloheimo and H. Stubb, Journal of Biomaterials Science Polymer Ed., 1995, 7, 577-586.

38. J. Wünsche, Y. Deng, P. Kumar, E. Di Mauro, E. Josberger, J. Sayago, A. Pezzella, F. Soavi, F. Cicoira, M. Rolandi and C. Santanto, Chemistry of Materials, 2015, 27, 436442.

39. M. R. Powell and B. Rosenberg, Bioenergetics, 1970, 1, 493-509.

40. A. J. Clulow, A. B. Mostert, M. Sheliakina, A. Nelson, N. Booth, P. L. Burn, I. R. Gentle and P. Meredith, Soft Matter, 2017, 13, 3954-3965.

41. M. M. Jastrzebska, H. Isotalo, J. Paloheimo, H. Stubb and B. Pilawa, Journal of Biomaterials Science Polymer Ed., 1996, 7, 781-793.

42. J. Rivnay, S. Inal, A. Salleo, R. M. Owens, M. Berggren and G. G. Malliaras, Nature Reviews Materials, 2018, 3, 17086.

43. S. Richert, I. Kuprov, M. D. Peeks, E. A. Suturina, J. Cremers, H. L. Anderson and C.

R. Timmel, Physical Chemistry Chemical Physics, 2017, 19, 16057.

44. A. Batagin-Neto, E. S. Bronze-Uhle and C. F. O. Graeff, Phys. Chem. Chem. Phys., 2015, 17, 7264-7274.

45. F. J. Grady and D. C. Borg, Journal of the American Chemical Society, 1968, 90, 2949-2952.

46. J. V. Paulin, A. Batagin-Neto and C. F. O. Graeff, Journal of Physical Chemistry B, 2019, 123, 1248-1255.

47. M. d'Ischia, A. Napolitano, A. Pezzella, P. Meredith and M. Buehler, Angewandte Chemie, 2019, 10.1002/ange.201914276.

48. M. d'Ischia, A. Napolitano, V. Ball, C. T. Chen and M. J. Buehler, Accounts of Chemical Research, 2014, 47, 3541-3550.

49. Y. Liu, K. Ai and L. Lu, Chemical Reviews, 2014, 114, 5057-5115. 